\title{
Pré-processamento do problema de cobertura de conjunto aplicado ao escalonamento de condutores
}

\author{
Ademir Aparecido Constantino ${ }^{1}$ \\ Edilson Costa de Castro ${ }^{1}$ \\ Silvio Alexandre de Araujo ${ }^{2}$ \\ Cândido Ferreira Xavier de Mendonça Neto ${ }^{3}$
}

\begin{abstract}
Resumo: o problema de escalonamento de condutores (PEC) consiste em distribuir de maneira eficiente o quadro de viagens de uma empresa de transporte coletivo entre os condutores disponíveis. Esse problema é comumente modelado como um problema de cobertura de conjunto - PCC (set covering problem). Nesse caso, um bom resultado para o PEC depende de uma boa construção e resolução do PCC. Porém, a maior parte da bibliografia relacionada trata apenas da resolução das instâncias do PCC, sem avaliar a influência dos procedimentos com sua construção, aqui denominado de pré-processamento. Este trabalho propõe-se e investigar metodologias heurísticas baseadas em Simulated Annealing para o pré-processamento de instâncias do PCC, cujas características possibilitem os algoritmos de resolução obterem melhores resultados para o PEC. Nos testes efetuados, conseguiu-se uma redução de até $8 \%$ no custo das soluções apresentadas, em comparação com a resolução de instâncias geradas por um método clássico de geração do PCC.
\end{abstract}

Palavras-chave: Escalonamento de condutores. Heurística. Simulated Annealing.

\begin{abstract}
: the crew scheduling problem consists on efficiently distribute trips of an urban transport company to available drivers. Generally it is modeled as a set covering problem (SCP). In this case, good results for the crew scheduling problem depend on good generation and resolution to the SCP. Most of works in this area proposes only SCP resolution methods, but does not include the SCP generation in their matters. This paper investigates and proposes heuristic methodologies based on Simulated Annealing to generate SCP instances, whose characteristics allow solution algorithms to obtain better results. In executed tests, costs were reduced up to 8\%, in comparison with a classical method of SCP generation.
\end{abstract}

Keywords: Driver scheduling. Heuristic. Simulated Annealing.

\section{Introdução}

A resolução do problema de planejamento de escala de veículos e condutores consiste em determinar um plano de distribuição de viagens sobre um conjunto de veículos e motoristas envolvidos na operação, ao longo de um período de planejamento. Como resultado desse processo, para cada veículo é alocado um conjunto de viagens a ser realizado e cada viagem é alocada a um condutor.

\footnotetext{
${ }^{1}$ Universidade Estadual de Maringá, Departamento de Informática, Av. Colombo, 5.790, Maringá (PR) — Brasil

\{ademir, eccastro@din.uem.br\}

${ }^{2}$ Universidade Estadual Paulista, Departamento de Ciências da Computação e Estatística, São José do Rio Preto (SP) Brasil

\{saraujo@ibilce.unesp.br\}

${ }^{3}$ Universidade de São Paulo, Escola de Artes Ciências e Humanidades, São Paulo (SP) — Brasil

\{cfxavier@usp.br\}
}

http://dx.doi.org/10.5335/rbca.v8i2.5131

Revista Brasileira de Computação Aplicada (ISSN 2176-6649), Passo Fundo, v. 8, n. 2, p. 28-43, jul. 201628 
O planejamento de escala de veículos e condutores responde a uma série de questões que são comuns em uma empresa de transporte rodoviário de passageiros, por exemplo: número de veículos necessários; viagens que devem ser realizadas por determinado veículo; decisões sobre o abastecimento dos veículos; número de condutores necessários; início e fim de cada turno de trabalho; dias de trabalho e de folga de cada condutor; viagens que cada condutor deve cumprir para cada dia; dente outras.

Este trabalho trata apenas do problema de escalonamento de condutores (PEC), que pode ser dividido em dois subproblemas: o escalonamento diário (enfoque deste trabalho) e o escalonamento semanal/mensal. No escalonamento diário (ou crew scheduling), a carga diária de trabalho é distribuída em escalas diárias de condutores, determinando-se, assim, quantos condutores serão necessários a cada dia e quais viagens fazem parte de cada escala. O escalonamento semanal/mensal (crew rostering) define os condutores que cumprirão cada uma dessas escalas diárias ao longo de uma semana ou mês, determinando, por exemplo, as folgas semanais de cada condutor.

O custo com pessoal em uma empresa de transporte representa aproximadamente $50 \%$ dos custos da empresa [1], portanto, a atividade de escalonamento de condutores não é a única atividade responsável por reduzir os custos da empresa. De acordo com [2] o problema operacional geral da empresa é dividido em pelo menos três subproblemas resolvidos sequencialmente: Escalonamento de veículos, escalonamento (diário) de condutores (crew scheduling) e escalonamento (de longo prazo) de condutores (crew rostering). É de conhecimento que a resolução ótima isolada de cada um desses problemas não garante a solução ótima do problema geral. Sendo assim, existem pesquisas direcionadas para a resolução integrada do PEC e do problema de escalonamento de veículos [3]. Dada a complexidade do problema integrado e da sua resolução, as pesquisas ainda são insipientes.

Uma revisão bibliográfica de métodos de escalonamento de condutores pode ser encontrada em [4], cujos métodos são classificados em três categorias: métodos heurísticos e métodos exatos baseados em programação matemática. Alguns trabalhos nessa linha são: [5] (sistema TRACS), [6] (sistema INTERPLAN), [7] (sistema HOT) e [8] (sistema COMPACS). Segundo [4], embora muitos desses sistemas ainda estejam sendo usados em empresas do mundo todo, em termos de pesquisa acadêmica, essas metodologias já foram abandonadas. Com a melhoria dos recursos computacionais disponíveis, tornou-se possível a utilização da programação matemática como metodologia para o escalonamento de condutores, especialmente a partir dos anos 1980. Com essa nova abordagem, modelos matemáticos começaram a ser usados para esse problema, sendo mais comumente usado o modelo do problema de cobertura de conjunto (PCC), apresentado na próxima seção. Por ser um problema que não admite resolução em tempo polinomial, surgiram muitos trabalhos que resolvem o PCC usando algoritmos heurísticos [9], [10] e [11]. Além disso, algumas meta-heurísticas também foram aplicadas, tais como: algoritmos genéticos e variantes [9], [12], [13], [14] e [15]; Ant System [16]; Busca Tabu [17] e [18]. Métodos exatos para o PCC relacionado ao PEC também podem ser encontrados na literatura. O trabalho de [1] apresenta um algoritmo híbrido baseado em programação matemática e programação por restrição. Nesse caso, os dados de cada linha de ônibus são separados e o PEC é resolvido para cada uma dessas linhas individualmente. São consideradas duas linhas de ônibus distintas, uma com 125 e outra 266 viagens com dois pontos de possíveis trocas de condutores. O trabalho resolve o PEC de cada linha da rota de ônibus usando o PCC de custo unitário, pois o objetivo é minimizar o número de condutores.

Apesar de o PCC ser um dos modelos mais empregados na tentativa de resolução do PEC, os trabalhos encontrados na literatura não têm explorado técnicas de como gerar boas instâncias do PCC antes da sua resolução, aqui denominado de pré-processamento do PCC. Não foi encontrado outro trabalho na literatura que investigasse esse pré-processamento do PCC aplicado ao PEC. Este trabalho mostra que essa atividade tem um papel importante para melhorar a solução do PEC via PCC. Assim, o principal objetivo deste trabalho é contribuir para o preenchimento dessa lacuna na literatura, apresentando o estudo de duas versões de um algoritmo heurístico para uma fase intermediária entre o escalonamento de veículos e de escalonamento de condutores (utilizando o PCC como modelo de resolução). A Figura 1 ilustra essa integração e a relação com o problema de pré-processamento, em que o pré-processamento compreende a geração de instância do PCC para que a seja resolvida na fase seguinte.

Para a resolução do PEC foram consideradas três instâncias de dados de duas empresas distintas. As duas maiores instâncias apresentam 602 e 657 viagens cada uma. Além disso, todos os pontos finais são considerados como possíveis pontos de troca de condutores, como também são consideradas as possibilidades de os 
condutores serem alocados em diferentes linhas de ônibus. Esses dois aspectos tornam o PEC difícil de ser resolvido por algoritmo exato.

O presente artigo está dividido nas seguintes seções: além da Introdução, tem-se, na seção 2, uma descrição do problema de cobertura de conjunto. Na seção 3 analisa-se o pré-processamento para o problema de cobertura de conjunto. Na seção 4 são apresentados os métodos de resolução do problema de escalonamento de condutores. Os resultados computacionais são apresentados na seção 5 e, na seção 6 , têm-se as conclusões e algumas propostas de trabalhos futuros.

Figura 1: Relação do escalonamento de veículos e de condutores com o pré-processamento do PCC

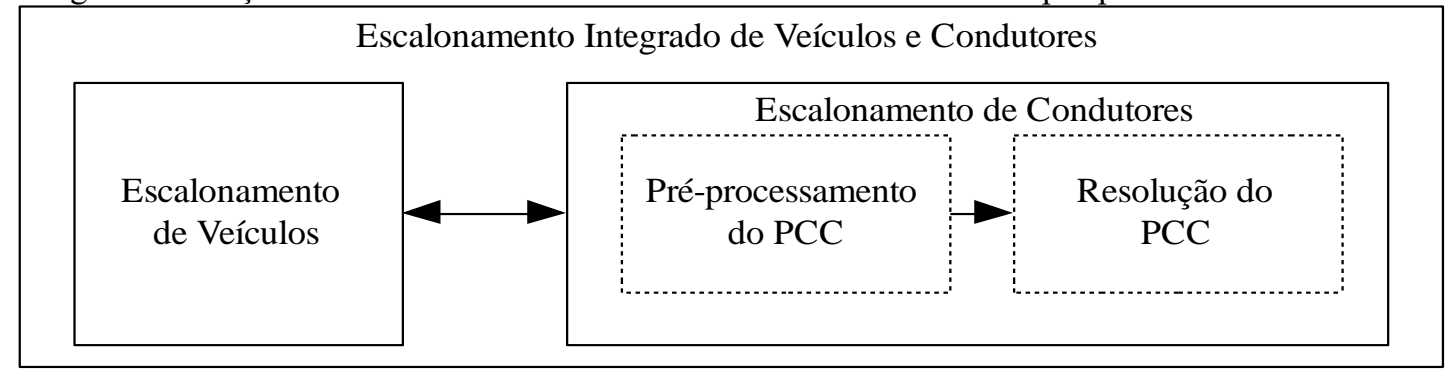

Fonte: elaborado pelos autores

\section{Problema de cobertura de conjunto}

Grande parte dos artigos encontrados na literatura modela o problema de escalonamento de condutores como um problema de cobertura de conjunto (PCC). A formulação matemática do PCC é a seguinte:

$$
\begin{gathered}
\operatorname{Min} \sum_{j \in J} c_{j} x_{j} \\
\text { s. a. } \sum_{j \in J} a_{i j} x_{j} \geq 1 \quad i \in I \\
x_{j} \in\{0,1\} \quad j \in J
\end{gathered}
$$

Sendo $I$ o conjunto de linhas a serem cobertas; $J$ é o conjunto de colunas para cobertura das linhas; $c_{j}$ é o custo da coluna; $j, a_{i j}=1$ se a coluna $j$ cobre a linha $i$ e zero caso contrário. No caso do escalonamento de condutores, as linhas da matriz representam as viagens a serem executadas e cada coluna representa uma - possibilidade de escala de trabalho de um condutor, que cobre (executa) uma pequena quantidade de linhas (viagens) do problema. Essas escalas são geradas a partir da enumeração completa de todas as combinações entre as linhas, que respeitem as restrições adotadas (sobreposição de horários, leis trabalhistas, regras da empresa e outros indicadores). Assim, a mesma linha pode ser coberta por várias colunas no modelo, sendo que a melhor solução consiste no subconjunto de colunas que cobre cada uma das linhas ao menos uma vez, com o menor custo possível.

$\mathrm{O}$ custo $c_{j}$, normalmente, corresponde ao valor pago, pela empresa, pelo cumprimento da escala, podendo variar, por exemplo, de acordo com a quantidade de horas extras contidas na escala. Outras variáveis podem entrar no cálculo de $c_{j}$, dependendo da modelagem que se queira adotar. Há casos em que o objetivo é reduzir o número de condutores, assim, nesses casos, considera-se custo unitário [1], ou seja, $c_{j}=1, \forall j \in J$.

A enumeração completa de todas as escalas possíveis, pela combinação das viagens, pode resultar em um número muito grande de colunas para o PCC. Uma estratégia encontrada na literatura para reduzir a dimensão do problema consiste em agrupar as viagens em partições de trabalho [8][19][2]. Por definição, uma partição de trabalho é um conjunto de viagens a serem executadas pelo mesmo condutor e com o mesmo veículo. Assim, o conjunto $I$ passa a representar um conjunto de partições de trabalho em vez de um conjunto de viagens. Essa estratégia reduz o número de linhas da matriz $\left[a_{i j}\right]$ e, consequentemente, o número de colunas. Tais partições de trabalho são resultados da combinação de conjunto de viagens extraídos das escalas de veículos. Algumas estratégias para a formação dessas partições são apresentadas na seção 3.1 . 


\section{Pré-processamento do problema de cobertura de conjunto}

A resolução do PEC modelado como um PCC compreende duas fases: pré-processamento, responsável pela construção das instâncias do PCC, e resolução das instâncias do PCC. Este artigo constata que um bom resultado para o PEC depende tanto de uma boa construção como da solução do PCC. Porém, a maior parte da bibliografia relacionada com o assunto trata apenas da segunda fase, ou seja, da investigação de técnicas computacionais para a resolução das instâncias do PCC, enquanto que a fase de construção das instâncias do PCC é negligenciada. São raros os casos da literatura que relatam o procedimento de construção do PCC. Quando relatam, utilizam procedimentos heurísticos bastante simples, por exemplo, limitam empiricamente o número de colunas geradas que cobrem determinadas linhas (partições), cujo propósito é simplesmente limitar a dimensão do PCC gerado para reduzir o esforço computacional na resolução. Este artigo investiga um procedimento heurístico para a fase de construção do PCC, aqui denominada de pré-processamento do PCC. Essa fase pode ser dividida em duas etapas: a divisão das escalas de veículos em partições de trabalho (seção 3.1) e a combinação dessas partições em possibilidades de escalas de condutor (seção 3.2).

\subsection{Divisão das escalas de veículos em partições de trabalho}

O desafio é descobrir qual é o melhor conjunto de partições de trabalho a ser extraídas da sequência de viagens diárias de cada veículo. O trabalho de [9] utiliza, para cada escala de veículo, um grafo de formação de partições de trabalho $H(I, C)$, em que o conjunto $I$ de vértices corresponde aos intervalos de tempo (possivelmente nulos) entre cada par de viagens consecutivas que formam a escala, além dos pontos $s$ e $t$, que representam, respectivamente, o início e o fim da mesma. Esta metodologia se assemelha a uma proposta encontrada em [19]. Este procedimento é uma forma heurística de combinar (agrupar) as viagens e com isso reduzir o número de colunas do PCC. Mas como se trata de um procedimento determinístico, este artigo utiliza essa ideia para criar um procedimento guloso randômico com o propósito de gerar alternativas que permitam uma exploração pelo algoritmo Simulated Annealing.

O grafo formado é acíclico, com um vértice de origem e um vértice terminal. A existência de uma aresta $\left(i_{x}\right.$ ,$\left.i_{y}\right) \in C$ indica a possibilidade de se formar uma partição de trabalho com as viagens entre os intervalos $i_{x} \mathrm{e} i_{y}$. $\mathrm{O}$ custo $c_{x y}$ associado a cada arco é relativo ao intervalo de trabalho $t_{x y}$ compreendido entre os intervalos $i_{x} \mathrm{e} i_{y}$. Assim, $c_{x y}$ é dado por:

$$
c_{x y}=\left\{\begin{array}{l}
P_{\min }, \text { se } t_{x y}<P_{\min } \\
\infty, \text { se } t_{x y}>P_{\text {máx }} \\
t_{x y}, \text { nos demais casos }
\end{array}\right.
$$

em que $P_{\min }$ e $P_{\max }$ são, respectivamente, os limites mínimo e máximo de comprimento das partições de trabalho, estabelecidos pelo usuário. Observe-se que é permitido que uma partição de trabalho tenha um comprimento menor que $P_{\min }$; embora, sua utilização nas escalas é dificultada pelo artifício de lhes ser atribuídas um custo maior do que seu comprimento (no caso, o próprio parâmetro $P_{\min }$ ). A Figura 2 apresenta um exemplo do grafo de formação de partições de trabalho para uma escala de cinco viagens.

Figura 2: Exemplo de um grafo de formação de partições de trabalho

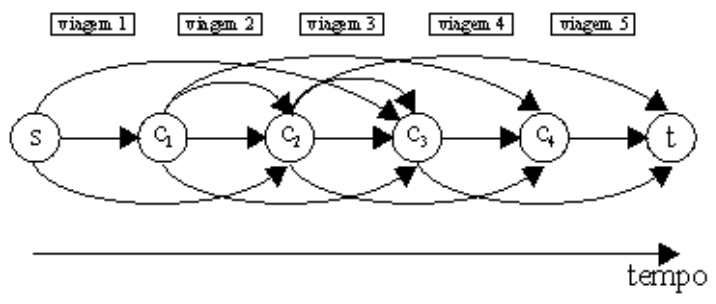

Fonte: [9]

Encontrar a melhor divisão de partições de trabalho para a escala em questão seria equivalente a encontrar o caminho de custo mínimo no grafo $H$, iniciando no vértice $s$ e finalizando no vértice $t$. Esse é um problema clássico em grafos e um algoritmo mais conhecido para esse caso é o algoritmo de Dijkstra [20]. O objetivo 
principal é dividir as escalas de veículos em partições de trabalho, ou seja, particionar as escalas de veículos nos pontos de maior intervalo entre as viagens, evitando, assim, que esses intervalos tornem-se períodos ociosos dentro das escalas de condutores.

Nos experimentos realizados por esse procedimento para construir o PCC, notou-se que algumas partições de trabalho eram cobertas por um número muito reduzido de colunas. Inclusive, se não for dada a oportunidade de formação de escalas de condutor com apenas uma partição de trabalho (o que ocorre em [9]) algumas partições poderão não ser cobertas por nenhuma coluna, inviabilizando o processo de escalonamento.

Assim, considerando que o algoritmo de Dijkstra é determinístico, este artigo propõe o uso de um algoritmo guloso randomizado que será utilizado na próxima seção em conjunto com o Simulated Annealing. O algoritmo, denominado ParticaoRandomica, é descrito a seguir:

\section{ALGORITMO 1: ParticaoRandomica}

Entrada: escala de um veículo;

Saída: sequência de vértices (que definirá conjunto de partições de trabalho);

Construir o grafo de formação de partições $H$;

$v=s$; //Iniciar com o vértice $s$ do grafo $H$;

Enquanto o vértice $v$ for diferente de $t$ faça

$v=$ vértice adjacente a $v$, selecionado aleatoriamente com probabilidade inversamente proporcional à distância.

A sequência de vértices escolhidos definirá a divisão da escala de um veículo, gerando um conjunto de partições de trabalho. Essas partições servirão de solução inicial, que passarão por melhorias por meio de um algoritmo de busca local que será descrito na seção 4 .

\subsection{Combinação das partições em escalas de condutores}

Com as partições definidas, o próximo passo é combiná-las, formando possíveis escalas de trabalho de condutores que representa uma coluna do PCC. O número de escalas possíveis (representadas por colunas no PCC) pode ser muito elevado, tendo em vista que cada escala é uma possível combinação de partições de trabalho. Para reduzir o tamanho das instâncias (linhas e colunas) do problema, alguns trabalhos da literatura aplicam métodos heurísticos para gerar apenas um subconjunto dessas colunas.

A forma mais comum para gerar essas colunas é o agrupamento dessas partições de maneira determinística seguindo algumas regras de formação impostas pela empresa. Normalmente uma escala de condutor é formada pela combinação de uma, duas, três ou até quatro partições, e deve obedecer aos seguintes parâmetros (referentes às leis trabalhistas e/ou às certas regras internas da empresa), ilustrados na Figura 3.

Figura 3: Parâmetros para a formação de escalas de condutores

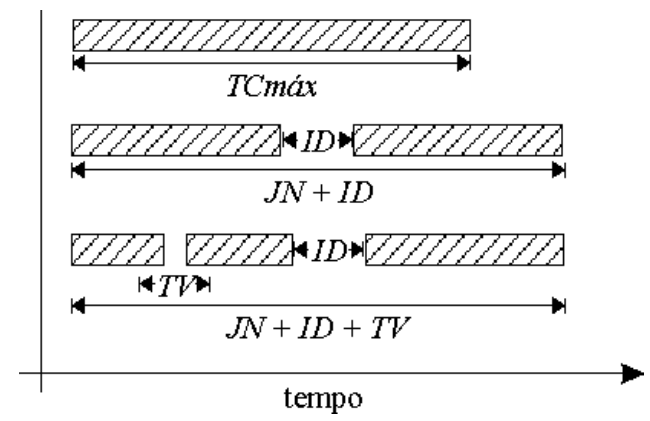

Fonte: elaborado pelos autores 
Sendo TCmax (trabalho contínuo máximo) o período de tempo máximo que um condutor pode trabalhar sem ter um período de descanso; $J N$ (jornada normal de trabalho) a duração máxima de uma escala de trabalho normal (sem horas extras); $I D_{\min }$ e $I D_{\max }$ (mínimo e máximo intervalo de descanso) delimitam o comprimento de um período de descanso (não é contabilizado como parte da jornada de trabalho); $T V$ (mínimo intervalo para troca de veículo) o tempo mínimo estimado para um condutor trocar de veículo entre uma partição de trabalho e outra (é contabilizado como parte da jornada de trabalho) e $H E$ (máximo período de horas extras) a quantidade máxima de horas extras que uma escala pode conter. Uma escala não pode ter duração maior do que $J N+H E$.

\section{Algoritmo para o pré-processamento}

A meta principal desta pesquisa é investigar algumas formas de melhorar a formação de partições com objetivo de obter soluções melhores para o PEC a partir da resolução do PCC. Para isto, são propostos dois algoritmos para o pré-processamento baseados na meta-heurística Simulated Annealing (SA) [21][22].

Os algoritmos propostos são iniciados com uma solução obtida pelo algoritmo ParticaoRandomica para cada uma das escalas de veículos. Os algoritmos fazem modificações no conjunto de partições por meio de uma estrutura de vizinhança, buscando otimizar uma função objetivo. Essas modificações geram novas partições de trabalho. Uma descrição geral utilizada para os dois algoritmos é dada a seguir.

Os parâmetros utilizados pelo algoritmo geral são:

$M T$ : tempo de execução utilizado como critério de parada do algoritmo;

$T$ : temperatura inicial;

$T L$ : número de iterações para cada valor da temperatura $(T)$ e

$C F$ : fator de resfriamento (decréscimo da temperatura a cada $T L$ iterações).

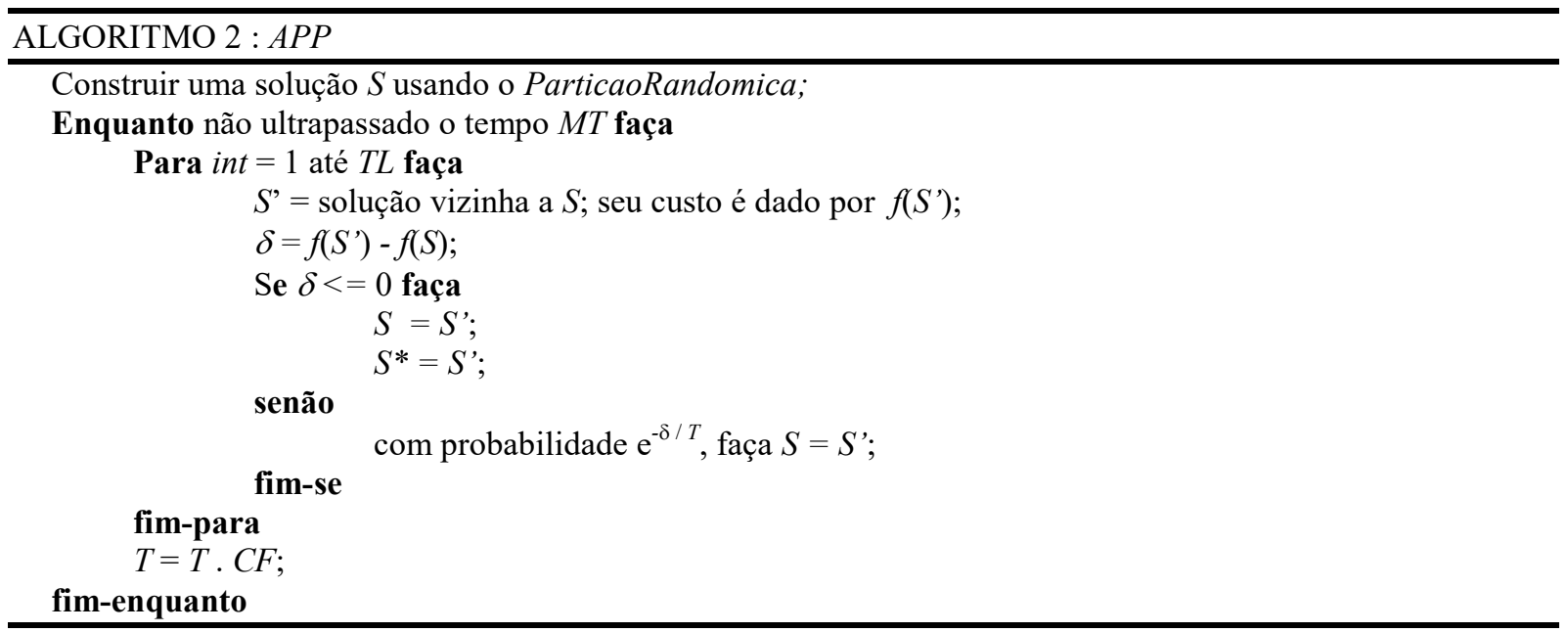

A seguir, são apresentados alguns passos importantes para a utilização da meta-heurística Simulated Annealing tais como: geração da solução inicial, geração da solução vizinha e cálculo da função $f$ (custo da solução).

Considere as seguintes notações: $V=\left\{v_{1}, v_{2}, \ldots, v_{n}\right\}$ o conjunto de viagens, sendo $n$ o número de viagens; $U_{i}$ $\subseteq V, i=1, \ldots, u$, escala do veículo $i$ para um número de $u$ veículos, sendo que $\bigcap_{i=1}^{u} U_{i}=\phi$ (as escalas não possuem viagens em comum) e $\bigcup_{i=1}^{u} U_{i}=V$ (a união de todas as escalas cobre todas as viagens). Considere $P_{i j}$ $\subseteq U_{i}, i=1, \ldots, u, j=1, \ldots, p_{i}$, sendo a partição $j$ da escala do veículo $i$, de forma que $\bigcap_{j=1}^{p_{i}} P_{i j}=\phi$ (as partições são distintas) e $\bigcup_{j=1}^{p_{i}} P_{i j}=U_{i}$ (a união das partições cobrem todas as viagens do veículo $i$ ). 
Os dados de entrada para o algoritmo $A P P$ são as escalas de veículos, portanto, foi considerada a existência dessas escalas. Define-se uma solução $S$ como sendo um conjunto contendo todas as partições $P_{i j}$ resultantes da divisão de todas as escalas de veículos $U_{i}, i=1, \ldots, u, j=1, \ldots, p_{i}$.

\subsection{Função de avaliação}

Para o cálculo da função de avaliação é necessário construir a instância do PCC correspondente à solução $S$ (uma solução $S$ é um conjunto de escalas de veículos com todas as suas escalas divididas em partições de trabalho). Por sua vez, para construir a instância do PCC é necessário enumerar todas as alternativas de escala de condutor possíveis usando as partições da solução $S$.

Todas as combinações possíveis de 1, 2, 3 e 4 partições são testadas na geração de escalas de trabalho. Porém, em razão das restrições e da possível sobreposição de horários, nem toda combinação de partições é capaz de gerar uma escala.

Depois de gerado o conjunto de alternativas de escalas de condutores (colunas do PCC), o custo de uma solução $S$ pode ser calculado, é quando se determina o valor da função de avaliação. Foram implementadas duas variantes para o cálculo da função de avaliação, gerando os algoritmos APP1 e APP2, tomando como base o algoritmo $A P P$ apresentado anteriormente.

\subsubsection{Algoritmo de pré-processamento 1}

A primeira variante busca por um PCC cuja linhas estejam cobertas pelo maior número de colunas, e da maneira mais homogênea possível. Assim, as instâncias do PCC que são geradas durante a execução do algoritmo são avaliadas de acordo com essa cobertura e homogeneidade e a função de avaliação é calculada da seguinte forma:

Seja $\operatorname{cob}(P)$ o número de colunas que cobrem a partição $P \in S$. Então:

$$
f(S)=\operatorname{col}(S) \times \frac{\delta(S)}{\mu(S)^{2}}
$$

sendo $\operatorname{col}(S)$ o número de colunas do problema; $\delta(S)$ e $\mu(S)$ são, respectivamente, a média e o desvio padrão de $\operatorname{cob}(P)$.

Ao minimizar o valor de $f(S)$, o algoritmo APP1 estará procurando por uma instância do PCC, em que cada linha é coberta pelo maior número de colunas possível $(\mu(S))$, de forma homogênea $(\delta(S))$ e sem aumentar desnecessariamente o número de colunas $(\operatorname{col}(\mathrm{S}))$.

\subsubsection{Algoritmo de pré-processamento 2}

A segunda variante avalia as instâncias do PCC geradas utilizando um método guloso de resolução, cujo custo da solução obtida é utilizado como uma estimativa da qualidade dessa solução. Assim, o PCC construído é submetido a um algoritmo heurístico construtivo com o objetivo de obter uma estimativa do custo da solução rapidamente. Nesse caso foi utilizado o algoritmo de [23]. Dessa forma, $f(S)$ é a soma dos custos das colunas que participam da solução construída para o PCC.

\subsection{Geração de uma solução vizinha}

Em ambas as variantes do algoritmo, para gerar uma solução vizinha $S^{\prime}$, é necessário, em primeiro lugar, selecionar-se uma partição $P$ da solução atual $S$ para ser modificada. A partição selecionada é então submetida a sete diferentes heurísticas de alteração, que dão origem a sete novas soluções. Finalmente, uma dessas soluções é selecionada aleatoriamente como sendo a solução vizinha $S$, com probabilidades de seleção inversamente proporcionais aos seus respectivos custos. A seguir, esse procedimento é descrito com mais detalhes. 


\subsubsection{Seleção da partição}

Seguem as formas de selecionar a partição:

- APP1: na primeira variante do algoritmo, a seleção da partição $P$ a ser modificada leva em consideração a instância do PCC criado a partir da solução atual $S$. A seleção é feita com base nos valores de $\operatorname{cob}(P)$ para as partições $P \in S$. Selecionam-se aleatoriamente duas partições, das quais aquela com maior $\mid \operatorname{cob}(P)$ - $\mu(S) \mid$ (abordagem tournament) será a partição escolhida para sofrer a modificação.

- APP2: para a segunda variante do algoritmo, é selecionada aleatoriamente uma partição com distribuição uniforme.

\subsubsection{Modificação da partição}

Após a seleção da partição $P$, ela é submetida a sete heurísticas de modificação. Sendo $U_{i}$ a escala de veículo que contém a partição $P$. As heurísticas são as seguintes:

- Expansão do limite inferior: caso exista uma partição $P^{\prime} \in U_{i}$ anterior a $P$, a última viagem de $P^{\prime}$ será transferida para a partição $P$. O processo se repete até que o local de origem da viagem transferida seja um ponto de troca de condutores.

- Retração do limite inferior: caso exista uma partição $P^{\prime} \in U_{i}$ anterior a $P$, a primeira viagem de $P$ será transferida para a partição $P$. O processo se repete até que o local de origem da viagem transferida seja um ponto de troca de condutores.

- Expansão do limite superior: caso exista uma partição $P^{\prime} \in U_{i}$ posterior a $P$, a primeira viagem de $P^{\prime}$ será transferida para a partição $P$. O processo se repete até que o local de origem da viagem transferida seja um ponto de troca de condutores.

- Retração do limite superior: caso exista uma partição $P^{\prime} \in U_{i}$ posterior a $P$, a última viagem de $P^{\prime}$ será transferida para a partição $P$. O processo se repete até que o local de origem da viagem transferida seja um ponto de troca de condutores.

- Divisão: seja $k$ o número de oportunidades de troca existentes dentro da partição $P$ (excluindo-se seu início e fim). Se $k>1$, divide-se a partição $P$ em duas novas partições no ponto da $n$-ésima oportunidade de troca. $\mathrm{O}$ valor de $n$ é dado $\lfloor\mathrm{k} / 2\rfloor$.

- União: une-se a partição $P$ a uma de suas partições vizinhas (caso exista o vizinho anterior e posterior, seleciona-se aleatoriamente um deles); caso essa união resulte em uma partição maior do que o permitido, transfere-se para $P$ apenas as viagens possíveis.

- Reconstrução das partições da escala: refaz-se toda a divisão em partições da escala $U_{i}$, utilizando o algoritmo guloso randomizado descrito no final da seção 3.1.

\subsection{Períodos ociosos dentro de escalas}

A utilização do algoritmo de Dijkstra para efetuar a divisão das escalas de veículos em partições faz com que essas partições contenham o mínimo possível de períodos ociosos, assim como as escalas de condutor que serão formadas a partir delas. A metodologia aqui proposta, ao procurar por novos conjuntos de partições, acaba efetuando uma relaxação nesta restrição. Para controlar essa relaxação, desenvolveu-se um mecanismo para evitar que períodos ociosos sejam incorporados excessivamente às partições de uma solução: seja $S_{0}$ a solução inicial do algoritmo, $t(p)$ a duração de uma partição $p$ qualquer; são descartadas automaticamente as soluções $S^{\prime}$ que apresentarem:

$$
\sum_{p^{\prime} \in S^{\prime}} t\left(p^{\prime}\right)>\sum_{p^{\prime} \in S_{0}} t(p)+\% p e r d a_{\max }
$$

sendo \%perda $a_{\max }$ um parâmetro de entrada definido para o algoritmo. 


\section{Resultados computacionais}

Nesta seção, são apresentados os resultados obtidos com a utilização das duas variantes do algoritmo de préprocessamento do PCC (APP1 e APP2) apresentadas na seção anterior. Para avaliação dos resultados, foram utilizadas três bases de dados distintas:

- As bases de dados de [9], correspondentes aos dados reais de uma empresa de transporte coletivo. Foram utilizados os quadros de viagens de dias úteis (602 viagens) e de domingos e feriados (212 viagens). Essas bases serão designadas por M602 e M212, respectivamente;

- A base de dados de uma empresa do estado do Paraná, com 657 viagens, referenciada como CV657.

Os testes foram efetuados em um computador equipado com processador AMD XP 2.7GH e 4 GB de memória RAM. Tanto APP1 quanto APP2 foram executados a partir de uma solução inicial obtida com o algoritmo ParticaoRandomica para efetuar a divisão das escalas de veículo. Assim, para efeito de comparação, os resultados obtidos com a aplicação de APP1 e APP2 serão confrontados com aqueles obtidos com a aplicação do algoritmo de Dijkstra.

As instâncias do PCC geradas pelas três abordagens (algoritmo de Dijkstra, APP1 e APP2) sobre as três bases de dados citadas (M602, M212 e CV657) foram submetidas a dois algoritmos genéticos cedidos por [24] e [25], designados aqui por AG1 e AG2, respectivamente. Esses algoritmos genéticos têm a função de resolver o PCC como uma forma de avaliar o resultado dos algoritmos propostos. Nos experimentos realizados foram utilizados os seguintes parâmetros:

AG1 - Tamanho da população: 1000 indivíduos; taxa de mutação: 3\%;

AG2 - Tamanho da população: 400 indivíduos; número de ciclos após convergência: 1000.

Os resultados para cada uma das três bases de dados são apresentados nas três seções seguintes. Em cada seção apresenta-se inicialmente a geração de uma única instância do PCC pelo algoritmo de Dijkstra e, em seguida, quatro diferentes resoluções do PCC com os algoritmos AG1 e AG2. Posteriormente, tem-se a geração de quatro diferentes instâncias do PCC com o algoritmo APP1, seguida de quatro diferentes resoluções para cada instância do PCC com os algoritmos AG1 e AG2. Por fim, repete-se o procedimento anterior gerando as instâncias do PCC com o algoritmo APP2. Os melhores resultados de cada tabela são destacados em negrito.

\subsection{Calibragem dos parâmetros}

Os parâmetros utilizados nos algoritmos APP1 e APP2 foram calibrados com base na observação da convergência e exploração do espaço de busca pelos algoritmos. Vários experimentos foram realizados para se chegar ao ajuste desses parâmetros.

A Figura 4 ilustra um exemplo dos algoritmos sobre base de dados CV657. O gráfico mostra uma convergência gradual dos algoritmos (cor cinza), enquanto que continuam explorando o espaço de soluções (cor preta) por meio do mecanismo de aceitação probabilística para soluções vizinhas pior que a incumbente. Esse é um aspecto importante para o desempenho de um algoritmo heurístico baseado em Simulated Annealing. 
Figura 4: Gráfico de convergência dos algoritmos APP1 e APP2
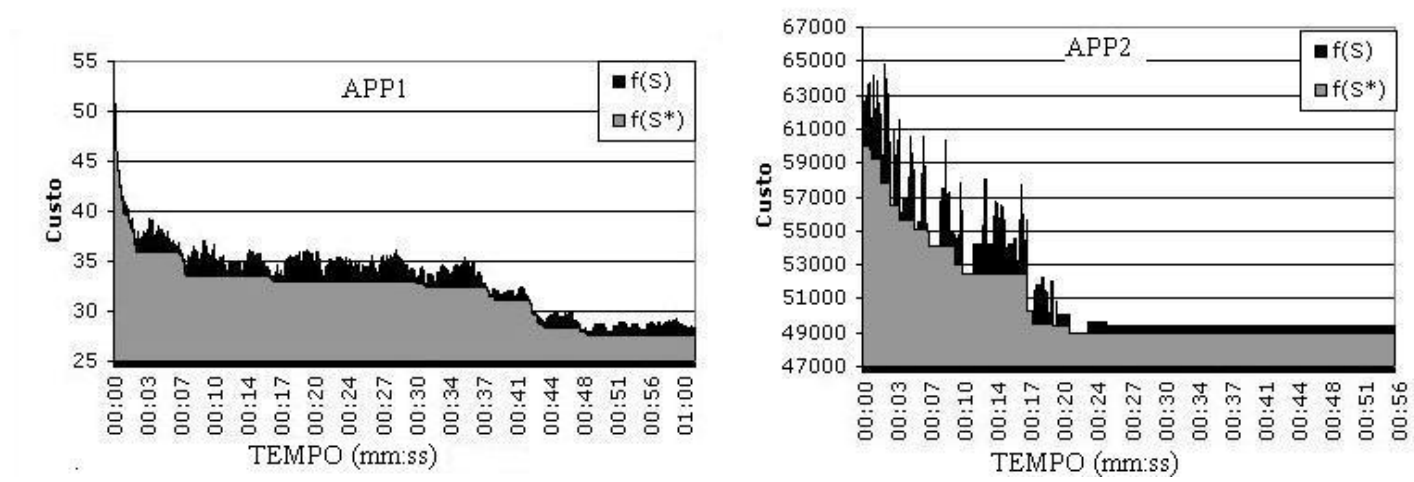

Fonte: elaborado pelos autores

\subsection{Experimento 1 - Resultados sobre a base de dados M602}

As tabelas a seguir resumem os parâmetros utilizados e os resultados. A coluna PCC significa o rótulo para o problema gerado na tabela, $M T$ é o tempo em segundos, partições é o número de partições (linhas) do problema gerado, escalas é o número de escalas (equivalentes ás colunas do PCC) do problema gerado e \%perda $a_{\max }$ é o valor para o parâmetro de entrada do algoritmo de pré-processamento.

Tabela 1: Características do PCC gerado para a instância M602

\begin{tabular}{|c|c|c|c|c|c|c|c|c|}
\hline \multirow{2}{*}{ Algoritmo } & \multirow{2}{*}{$P C C$} & \multicolumn{3}{|c|}{ Parametrização } & \multirow[b]{2}{*}{$M T$} & \multirow[b]{2}{*}{$\%$ perda $_{\max }$} & \multicolumn{2}{|c|}{ Resultados } \\
\hline & & $T$ & $C F$ & $T L$ & & & partições & escalas \\
\hline Dijkstra & 0.1 & - & - & - & - & - & 177 & 5451 \\
\hline \multirow{4}{*}{ APP1 } & 1.1 & 1000 & 0,95 & 40 & 60 & 0,05 & 179 & 6276 \\
\hline & 1.2 & 1000 & 0,95 & 40 & 60 & 0,15 & 178 & 5886 \\
\hline & 1.3 & 1000 & 0,95 & 120 & 240 & 0,25 & 178 & 5376 \\
\hline & 1.4 & 1000 & 0,95 & 120 & 60 & 0,25 & 177 & 5494 \\
\hline \multirow{4}{*}{ APP2 } & 2.1 & 500000 & 0,95 & 60 & 120 & 0,01 & 177 & 5453 \\
\hline & 2.2 & 500000 & 0,95 & 100 & 240 & 0,01 & 178 & 5383 \\
\hline & 2.3 & 500000 & 0,95 & 60 & 120 & 0,1 & 177 & 5616 \\
\hline & 2.4 & 500000 & 0,95 & 100 & 300 & 0,1 & 177 & 5611 \\
\hline
\end{tabular}

Fonte: elaborada pelos autores

As tabelas a seguir resumem resultados obtidos, sendo que $i t$. significa o número de iterações efetuadas pelo algoritmo, cond. o número de condutores utilizados na solução gerada e tempo dado em segundos. 
Tabela 2: Resolução do PCC para a instância M602

\begin{tabular}{|c|c|c|c|c|c|c|c|c|c|}
\hline \multirow{2}{*}{ Algoritmo } & \multirow{2}{*}{$P C C$} & \multicolumn{4}{|c|}{ AG1 } & \multicolumn{4}{|c|}{ AG2 } \\
\hline & & $i t$. & cond. & custo & tempo & it. & cond. & custo & tempo \\
\hline \multirow{4}{*}{ Dijkstra } & 0.1 & 54085 & 90 & 40048 & 155 & 44800 & 91 & 38408 & 125 \\
\hline & 0.1 & 55706 & 90 & 39787 & 161 & 43400 & 91 & 38435 & 70 \\
\hline & 0.1 & 53857 & 90 & 39912 & 169 & 47050 & 91 & 38439 & 112 \\
\hline & 0.1 & 51622 & 90 & 39823 & 149 & 44350 & 91 & 38414 & 49 \\
\hline \multirow{16}{*}{ APP1 } & 1.1 & 51596 & 89 & 39383 & 158 & 56950 & 90 & 38007 & 107 \\
\hline & 1.1 & 50954 & 89 & 39065 & 158 & 40500 & 90 & 38093 & 114 \\
\hline & 1.1 & 62585 & 90 & 39652 & 189 & 45450 & 91 & 38316 & 152 \\
\hline & 1.1 & 51574 & 89 & 39285 & 159 & 54150 & 90 & 38075 & 67 \\
\hline & 1.2 & 39731 & 90 & 39731 & 158 & 56350 & 91 & 38449 & 150 \\
\hline & 1.2 & 70044 & 90 & 39650 & 198 & 76750 & 91 & 38359 & 107 \\
\hline & 1.2 & 148124 & 89 & 38478 & 402 & 67700 & 91 & 38406 & 139 \\
\hline & 1.2 & 57103 & 90 & 39609 & 164 & 66930 & 91 & 84405 & 132 \\
\hline & 1.3 & 49615 & 89 & 40016 & 142 & 63200 & 90 & 38127 & 106 \\
\hline & 1.3 & 55779 & 89 & 39787 & 158 & 44250 & 90 & 38120 & 112 \\
\hline & 1.3 & 75717 & 89 & 39033 & 181 & 55350 & 91 & 38337 & 158 \\
\hline & 1.3 & 50240 & 89 & 39760 & 144 & 74800 & 90 & 38215 & 140 \\
\hline & 1.4 & 49782 & 89 & 39820 & 139 & 53800 & 90 & 38148 & 100 \\
\hline & 1.4 & 97099 & 89 & 38979 & 264 & 55450 & 89 & 37826 & 41 \\
\hline & 1.4 & 76299 & 89 & 39191 & 212 & 54000 & 90 & 38059 & 95 \\
\hline & 1.4 & 64415 & 89 & 39562 & 182 & 54450 & 90 & 38063 & 80 \\
\hline \multirow{16}{*}{ APP2 } & 2.1 & 56052 & 89 & 39814 & 160 & 56950 & 90 & 38007 & 107 \\
\hline & 2.1 & 58494 & 89 & 39125 & 162 & 40500 & 90 & 38093 & 114 \\
\hline & 2.1 & 82078 & 90 & 39297 & 236 & 45450 & 91 & 38316 & 152 \\
\hline & 2.1 & 56091 & 89 & 39069 & 165 & 54150 & 90 & 38075 & 67 \\
\hline & 2.2 & 61149 & 88 & 39307 & 174 & 56350 & 91 & 38449 & 150 \\
\hline & 2.2 & 70914 & 89 & 39156 & 208 & 76750 & 91 & 38359 & 107 \\
\hline & 2.2 & 54187 & 89 & 39953 & 165 & 67700 & 91 & 38406 & 139 \\
\hline & 2.2 & 53054 & 89 & 39977 & 161 & 66930 & 91 & 84405 & 132 \\
\hline & 2.3 & 51643 & 90 & 40153 & 160 & 63200 & 90 & 38127 & 106 \\
\hline & 2.3 & 49245 & 90 & 39845 & 146 & 44250 & 90 & 38120 & 112 \\
\hline & 2.3 & 38837 & 89 & 39735 & 106 & 55350 & 91 & 38337 & 158 \\
\hline & 2.3 & 63440 & 90 & 39491 & 192 & 74800 & 90 & 38215 & 140 \\
\hline & 2.4 & 58987 & 89 & 39028 & 161 & 53800 & 90 & 38148 & 100 \\
\hline & 2.4 & 48002 & 88 & 39659 & 135 & 55450 & 89 & 37826 & 41 \\
\hline & 2.4 & 68944 & 88 & 38886 & 189 & 54000 & 90 & 38059 & 95 \\
\hline & 2.4 & 55507 & 88 & 39038 & 138 & 54450 & 90 & 38063 & 80 \\
\hline
\end{tabular}

Fonte: elaborada pelos autores

Nos testes sobre a base de dados M602, verifica-se que ambos algoritmos (APP1 e APP2) conseguiram melhorar os resultados obtidos pelos algoritmos de resolução do PCC, AG1 e AG2 - em comparação com as instâncias do PCC geradas pelo algoritmo de Dijkstra. Isso pode ser notado pelas colunas custo, na qual estão destacados em negrito os melhores custos obtidos com os algoritmos Dijkstra, APP1 e APP2. O melhor custo do Dijkstra foi 38408 com AG2, enquanto que o menor custo para APP1 e APP2 foi 37826, usando AG2 para ambos. A redução do custo das soluções variou entre 1,5 e 3,3\%, além disso, nota-se a redução do número de até dois condutores, sendo noventa o menor número com Dijkstra e 88 o menor número com APP2.

\subsection{Experimento 2 - Resultados sobre a base de dados M212}

As tabelas 3 e 4 apresentam os resultados alcançados com essa instância. 
Tabela 3: Características do PCC gerado para a instância M212

\begin{tabular}{|c|c|c|c|c|c|c|c|c|}
\hline \multirow{2}{*}{ Algoritmo } & \multirow{2}{*}{$P C C$} & \multicolumn{3}{|c|}{ Parametrização } & \multirow[b]{2}{*}{$M T$} & \multirow[b]{2}{*}{$\%$ perda $_{\max }$} & \multicolumn{2}{|c|}{ Resultados } \\
\hline & & $T$ & $C F$ & $T L$ & & & partições & escalas \\
\hline Dijkstra & 0.1 & - & - & - & - & - & 66 & 528 \\
\hline \multirow{4}{*}{ APP1 } & 1.1 & 300 & 0,99 & 30 & 60 & 0,01 & 65 & 496 \\
\hline & 1.2 & 300 & 0,99 & 30 & 60 & 0,05 & 64 & 473 \\
\hline & 1.3 & 500 & 0,99 & 40 & 60 & 0,1 & 63 & 454 \\
\hline & 1.4 & 500 & 0,99 & 40 & 60 & 0,2 & 62 & 412 \\
\hline \multirow{4}{*}{ APP2 } & 2.1 & 200000 & 0,99 & 40 & 60 & 0,01 & 63 & 449 \\
\hline & 2.2 & 200000 & 0,99 & 40 & 60 & 0,05 & 63 & 444 \\
\hline & 2.3 & 200000 & 0,99 & 40 & 60 & 0,1 & 62 & 411 \\
\hline & 2.4 & 300000 & 0,99 & 40 & 60 & 0,1 & 62 & 434 \\
\hline
\end{tabular}

Fonte: elaborada pelos autores

Tabela4: Resolução do PCC para a instância M212

\begin{tabular}{|c|c|c|c|c|c|c|c|c|c|}
\hline \multirow{2}{*}{ Algoritmo } & \multirow{2}{*}{$P C C$} & \multicolumn{4}{|c|}{ AG1 } & \multicolumn{4}{|c|}{ AG2 } \\
\hline & & it. & cond. & custo & tempo & it. & cond. & custo & tempo \\
\hline \multirow{4}{*}{ Dijkstra } & 0.1 & 6194 & 33 & 14589 & 14 & 9150 & 33 & 14492 & 01 \\
\hline & 0.1 & 5803 & 33 & 14922 & 13 & 8150 & 33 & 14503 & 01 \\
\hline & 0.1 & 5849 & 33 & 14731 & 13 & 7300 & 33 & 14537 & 01 \\
\hline & 0.1 & 6195 & 33 & 14658 & 14 & 14600 & 33 & 14416 & 02 \\
\hline \multirow{16}{*}{ APP1 } & 1.1 & 5402 & 32 & 14934 & 12 & 13150 & 33 & 14547 & 01 \\
\hline & 1.1 & 4053 & 32 & 14692 & 09 & 17500 & 33 & 14453 & 02 \\
\hline & 1.1 & 4110 & 32 & 14377 & 09 & 11150 & 33 & 14570 & 01 \\
\hline & 1.1 & 5529 & 32 & 14510 & 13 & 10200 & 33 & 14608 & 01 \\
\hline & 1.2 & 4000 & 32 & 14614 & 12 & 20100 & 32 & 14426 & 02 \\
\hline & 1.2 & 5559 & 32 & 14708 & 12 & 15600 & 32 & 14488 & 02 \\
\hline & 1.2 & 5535 & 31 & 14359 & 15 & 18600 & 32 & 14392 & 02 \\
\hline & 1.2 & 3944 & 31 & 14422 & 04 & 15400 & 32 & 14454 & 02 \\
\hline & 1.3 & 5077 & 31 & 14493 & 11 & 11750 & 33 & 14543 & 01 \\
\hline & 1.3 & 5620 & 31 & 14715 & 12 & 11150 & 33 & 14556 & 01 \\
\hline & 1.3 & 5496 & 32 & 14469 & 12 & 13800 & 32 & 14362 & 02 \\
\hline & 1.3 & 4773 & 31 & 14576 & 10 & 16850 & 33 & 14535 & 02 \\
\hline & 1.4 & 5252 & 33 & 15023 & 12 & 13400 & 34 & 15076 & 01 \\
\hline & 1.4 & 7116 & 33 & 15163 & 16 & 11200 & 34 & 15071 & 01 \\
\hline & 1.4 & 4264 & 33 & 15269 & 10 & 18950 & 34 & 15023 & 02 \\
\hline & 1.4 & 4014 & 33 & 15418 & 09 & 10750 & 34 & 15072 & 01 \\
\hline \multirow{16}{*}{ APP2 } & 2.1 & 4135 & 31 & 14005 & 10 & 11650 & 31 & 13729 & 01 \\
\hline & 2.1 & 3408 & 31 & 13902 & 08 & 12500 & 31 & 13764 & 01 \\
\hline & 2.1 & 4081 & 31 & 13740 & 09 & 7850 & 31 & 13778 & 01 \\
\hline & 2.1 & 4954 & 31 & 14021 & 12 & 8650 & 31 & 13765 & 01 \\
\hline & 2.2 & 3691 & 32 & 14568 & 07 & 14250 & 32 & 14151 & 01 \\
\hline & 2.2 & 4308 & 31 & 14025 & 08 & 6950 & 32 & 14158 & 01 \\
\hline & 2.2 & 6243 & 32 & 14338 & 07 & 9650 & 32 & 14121 & 01 \\
\hline & 2.2 & 3406 & 31 & 14319 & 06 & 10050 & 32 & 14118 & 01 \\
\hline & 2.3 & 6476 & 31 & 14042 & 13 & 11150 & 31 & 13988 & 01 \\
\hline & 2.3 & 5489 & 32 & 14325 & 11 & 6200 & 32 & 14109 & 01 \\
\hline & 2.3 & 4603 & 31 & 14029 & 09 & 8350 & 31 & 14018 & 01 \\
\hline & 2.3 & 3625 & 31 & 14257 & 06 & 8400 & 31 & 14059 & 01 \\
\hline & 2.4 & 5541 & 31 & 14070 & 12 & 13450 & 31 & 13874 & 01 \\
\hline & 2.4 & 5566 & 31 & 14101 & 12 & 14450 & 31 & 13842 & 01 \\
\hline & 2.4 & 4451 & 31 & 14380 & 10 & 18350 & 31 & 13826 & 02 \\
\hline & 2.4 & 5868 & 31 & 14073 & 11 & 6900 & 31 & 13997 & 01 \\
\hline
\end{tabular}

Fonte: elaborada pelos autores 
Sobre a base de dados M212, verifica-se, também, que ambos os algoritmos (APP1 e APP2) conseguiram melhorar significativamente os resultados obtidos pelos algoritmos de resolução do PCC, AG1 e AG2 - em comparação com as instâncias do PCC geradas pelo algoritmo de Dijkstra. Isso pode ser observado nas colunas custo, em que estão destacados em negrito os melhores custos obtidos com os algoritmos Dijkstra, APP1 e APP2. O melhor custo do Dijkstra foi 14416 com AG2, enquanto que o menor custo com APP1 e APP2 foram 14359 (com AG1) e 13729 (com AG2), respectivamente. Portanto, a redução do custo das soluções chegou a $4,7 \%$, utilizando-se até dois condutores a menos como pode se constatar, com os menores valores para as colunas cond, sendo 33 com Dijkstra e 32 para APP1 e APP2.

\subsection{Experimento 3 - Resultados sobre a base de dados CV657}

As tabelas 5 e 6 exibe os resultados alcançados com a presente instância.

Tabela 5: Características do PCC gerado para a instância M657

\begin{tabular}{|c|c|c|c|c|c|c|c|c|}
\hline \multirow{2}{*}{ Algoritmo } & \multirow{2}{*}{$P C C$} & \multicolumn{5}{|c|}{ Parametrização } & \multicolumn{2}{|c|}{ Resultados } \\
\hline & & $T$ & $C F$ & $T L$ & $M T$ & $\%$ perda $_{\max }$ & partições & escalas \\
\hline Dijkstra & 0.1 & - & - & - & - & - & 125 & 1196 \\
\hline \multirow{4}{*}{ APP1 } & 1.1 & 600 & 0,95 & 60 & 60 & 0,01 & 139 & 2607 \\
\hline & 1.2 & 600 & 0,95 & 120 & 60 & 0,01 & 144 & 3473 \\
\hline & 1.3 & 600 & 0,95 & 60 & 60 & 0,1 & 144 & 3210 \\
\hline & 1.4 & 600 & 0,95 & 120 & 60 & 0,1 & 143 & 3227 \\
\hline \multirow{4}{*}{ APP2 } & 2.1 & 2000000 & 0,95 & 40 & 60 & 0,01 & 128 & 2040 \\
\hline & 2.2 & 2000000 & 0,95 & 40 & 60 & 0,05 & 138 & 3581 \\
\hline & 2.3 & 2000000 & 0,95 & 80 & 60 & 0,05 & 130 & 2840 \\
\hline & 2.4 & 2000000 & 0,95 & 60 & 60 & 0,1 & 132 & 2228 \\
\hline
\end{tabular}

Tabela 6: Resolução do PCC para a instância M657

\begin{tabular}{|c|c|c|c|c|c|c|c|c|c|}
\hline \multirow{2}{*}{ Algoritmo } & \multirow{2}{*}{$P C C$} & \multicolumn{4}{|c|}{ AG1 } & \multicolumn{4}{|c|}{ AG2 } \\
\hline & & it. & cond. & custo & teтро & it. & cond. & custo & tempo \\
\hline \multirow{4}{*}{ Dijkstra } & 0.1 & 18149 & 87 & 40090 & 19 & 17450 & 87 & 38983 & 18 \\
\hline & 0.1 & 20405 & 87 & 40220 & 19 & 21450 & 87 & 38983 & 20 \\
\hline & 0.1 & 19782 & 87 & 40056 & 36 & 19800 & 87 & 38983 & 18 \\
\hline & 0.1 & 20274 & 87 & 39964 & 37 & 22550 & 87 & 38983 & 16 \\
\hline \multirow{16}{*}{ APP1 } & 1.1 & 42155 & 83 & 38431 & 52 & 47700 & 83 & 37117 & 37 \\
\hline & 1.1 & 35107 & 83 & 38032 & 39 & 30500 & 83 & 37112 & 30 \\
\hline & 1.1 & 33957 & 83 & 38716 & 31 & 46300 & 83 & 37063 & 72 \\
\hline & 1.1 & 34137 & 83 & 38350 & 86 & 41350 & 83 & 37067 & 37 \\
\hline & 1.2 & 34646 & 81 & 37982 & 39 & 58050 & 82 & 36606 & 145 \\
\hline & 1.2 & 32644 & 82 & 37776 & 51 & 57150 & 82 & 36619 & 42 \\
\hline & 1.2 & 32799 & 81 & 37193 & 58 & 57150 & 82 & 36677 & 56 \\
\hline & 1.2 & 34426 & 82 & 37698 & 58 & 74600 & 82 & 36628 & 59 \\
\hline & 1.3 & 29958 & 81 & 37576 & 75 & 51650 & 82 & 36712 & 75 \\
\hline & 1.3 & 33257 & 81 & 37927 & 91 & 58100 & 82 & 36715 & 43 \\
\hline & 1.3 & 38911 & 81 & 37423 & 104 & 55250 & 81 & 36382 & 79 \\
\hline & 1.3 & 50550 & 81 & 37351 & 134 & 53850 & 81 & 36384 & 56 \\
\hline & 1.4 & 33729 & 81 & 37659 & 96 & 71750 & 81 & 36630 & 104 \\
\hline & 1.4 & 39209 & 81 & 37776 & 104 & 63100 & 81 & 36647 & 125 \\
\hline & 1.4 & 29074 & 81 & 37922 & 72 & 65750 & 81 & 36683 & 54 \\
\hline & 1.4 & 28587 & 81 & 38094 & 78 & 65150 & 81 & 36696 & 63 \\
\hline \multirow{5}{*}{ APP2 } & 2.1 & 20123 & 83 & 37658 & 47 & 25700 & 83 & 36900 & 29 \\
\hline & 2.1 & 23365 & 83 & 37487 & 54 & 18550 & 83 & 36952 & 33 \\
\hline & 2.1 & 30440 & 83 & 37488 & 70 & 18600 & 83 & 36957 & 44 \\
\hline & 2.1 & 22592 & 83 & 37831 & 53 & 21700 & 83 & 36952 & 40 \\
\hline & 2.2 & 24809 & 82 & 38145 & 60 & 21400 & 82 & 36738 & 53 \\
\hline
\end{tabular}




\begin{tabular}{ccccccccccc}
2.2 & 24102 & 82 & 38214 & 59 & 30600 & 82 & 36635 & 68 \\
\hline 2.2 & 32031 & 82 & 37606 & 77 & 27600 & 82 & 36680 & 67 \\
\hline 2.2 & 39510 & 82 & 37653 & 93 & 28250 & 82 & 36694 & 56 \\
\hline 2.3 & 19417 & 81 & 36833 & 48 & 25950 & 82 & 36384 & 59 \\
\hline 2.3 & 18886 & 81 & 37134 & 47 & 22250 & 81 & $\mathbf{3 6 0 7 4}$ & 25 \\
\hline 2.3 & 22093 & 81 & 37195 & 55 & 33600 & 82 & 36402 & 86 \\
\hline 2.3 & 23031 & 81 & 36677 & 57 & 24900 & 82 & 36402 & 29 \\
\hline 2.4 & 21906 & 83 & 38026 & 53 & 24650 & 83 & 37235 & 54 \\
\hline 2.4 & 24825 & 83 & 37779 & 65 & 25600 & 83 & 37235 & 40 \\
\hline 2.4 & 22421 & 83 & 38054 & 51 & 26100 & 83 & 37237 & 47 \\
\hline 2.4 & 22111 & 83 & 38609 & 50 & 26450 & 83 & 37234 & 60 \\
\hline
\end{tabular}

Fonte: elaborada pelos autores

Sobre a base de dados CV657, verifica-se que ambos algoritmos (APP1 e APP2) também conseguiram melhorar os resultados obtidos pelos algoritmos de resolução do PCC, AG1 e AG2 - em comparação com as instâncias do PCC geradas pelo algoritmo de Dijkstra. Considerando os melhores custos obtidos com os algoritmos Dijkstra, APP1 e APP2, destacados, em negrito, na coluna custo, nota-se que o melhor custo do Dijkstra foi 38983 com AG2, enquanto que o menor custo com APP1 e APP2 foram 36382 (com AG2) e 36074 (com AG2), respectivamente. A redução do custo das soluções chegou a $8,2 \%$, utilizando-se até seis condutores a menos, como pode ser notado na coluna cond., sendo 87 condutores com Dijkstra e 81 condutores com APP1 e APP2.

Nota-se pelos resultados apresentados que, ao contrário das instâncias geradas para as duas bases de teste anteriores, a quantidade de partições geradas pelo algoritmo de pré-processamento é maior em relação à instância gerada pelo algoritmo de Dijkstra. Em termos percentuais, o acréscimo no número de partições girou em torno de $15 \%$.

\section{Conclusões e trabalhos futuros}

Considerando que o problema de cobertura de conjunto é um dos modelos mais empregados para a resolução do problema de escalonamento de condutores, este artigo investigou o problema de pré-processamento do PCC que surge na resolução do PEC em empresas de transporte coletivo. Esse pré-processamento é praticamente negligenciado pela literatura, assim, este artigo propõe e investiga uma abordagem inédita para esta questão e constata-se a sua importância para obter soluções melhores para o PEC. Para isto, foram propostas e investigadas duas versões de algoritmos inspirados na meta-heurística Simulated Annealing, sendo APP1 e APP2, que utilizam um procedimento guloso randômico para encontrar partições alternativas iniciais e gerar as instâncias do PCC.

Os algoritmos foram testados com dados reais e de empresa de transporte de grande porte. As duas versões dos algoritmos apresentaram resultados melhores comparados com uma técnica da literatura que utiliza a obtenção de caminhos mínimos em grafos para a construção do PCC. A redução dos custos no PEC chegou até $8,2 \%$ para a maior instância analisada. Apesar de não haver outros algoritmos na literatura para comparações, os resultados indicam que a investigação do pré-processamento tem um papel importante para a redução dos custos operacionais no escalonamento de condutores quando modelado como um PCC. Considerando que o custo com pessoal em uma empresa de transporte representa aproximadamente $50 \%$ dos custos da empresa [1], nota-se que aplicação dessa abordagem de pré-processamento é vantajosa uma vez que o esforço computacional neste processo é baixo (poucos minutos de processamento) frente aos ganhos na qualidade das soluções.

Os algoritmos propostos, APP1 e APP2, utilizaram outros dois algoritmos, AG1 e AG2, que auxiliaram na resolução do PCC. No entanto, eles podem ser substituídos por outros algoritmos heurísticos, ou exatos da literatura, para a resolução do PCC. Considerando a hipótese de substituí-los por algoritmos mais eficientes e efetivos reforça a possibilidade de melhorar ainda mais os resultados alcançados pelos algoritmos de préprocessamento propostos, aumentando a credibilidade sobre os algoritmos APP1 e APP2. 
Por fim, destaca-se que não foram encontrados outros trabalhos na literatura que avalie o pré-processamento do PCC aplicado ao PEC. Assim, este trabalho também contribui para a cobertura dessa lacuna na literatura.

Como trabalhos futuros, os resultados obtidos neste trabalho incentivam a pesquisa de novas metodologias para o pré-processamento do PCC na resolução do PEC, como a exploração de outras meta-heurísticas e procedimentos de busca local. Além disso, esta abordagem de resolução com pré-processamento introduz uma nova possibilidade de resolução integrada do problema de escalonamento de veículos e de condutores, usando o PCC como modelo para o PEC, uma vez que pré-escalas de veículos poderiam ser avaliadas pelo préprocessamento.

\section{Referências}

[1] YUNES, T. H.; MOURA, A. V.; DE SOUZA, C. C. Hybrid column generation approaches for urban transit crew management problems. Transportation Science, Atlanta, GA. v. 39, n. 2, p. 273-288, 2005.

[2] BODIN, L.; GOLDEN, B.; ASSAD, A.; BALL, M. Routing and scheduling of vehicles and crews: the state of the art. Computers and Operations Research, Amsterdam, v. 10, n. 2, p. 63-21, 1983.

[3] FRELING R.; HUISMAN, D.; WAGELMANS, A. P. Models and algorithms for integration of vehicle and crew scheduling. Journal of Scheduling, New York, v. 6, n. 1, p. 63-85, 2003.

[4] LAYFIELD, C. A constraint programming pre-processor for duty scheduling. Tese (Doutorado em Ciência da Computação) - University of Leeds, England, 2002.

[5] PARKER, M. E.; SMITH, B. M. Two approaches to computer crew scheduling. In: A. Wren, Computer Scheduling of Public Transport, Amsterdam: Springer Verlag, 1981, p. 193-221.

[6] MOTT, P.;FRITSCHE, H. INTERPLAN — an interactive program system for crew scheduling and rostering of public transport. Computer-Aided Transit Scheduling, Berlin,: Springer Verlag, v. 308, n. 1, p. 200-211, 1988.

[7] DADUNA, J. R.; MOJSILOVIC, M. Computer-aided vehicle and duty scheduling using HOT programme system. Computer-Aided Transit Scheduling, Berlin,: Springer Verlag, v. 308, n. 1, p. 133-146, 1988.

[8] WREN, A.; FORES, S.; KWAN, A.; KWAN, R.; PARKER, M.; PROLL L. et al. A flexible system for scheduling drivers. Journal of Scheduling, London, v. 6, p. 437-455, 2003.

[9] MAYERLE, S. F. Um sistema de apoio à decisão para o planejamento operacional de empresas de transporte rodoviário urbano de passageiros. Tese (Doutorado em Engenharia de Produção) Universidade Federal de Santa Catarina, Florianópolis, 1996.

[10] SMITH, B. M. IMPACS - a bus crew scheduling system using integer programming. Matematical. Programming, Nova York, v. 42, n. 1, p. 181-187, 1988.

[11] LESSARD, R.; ROUSSEAU, J. M.; DUPUIS, D. HASTUS I: a mathematical programming approach to the bus driver scheduling problem. Computer Scheduling of Public Transport, Netherlands North Holland, p. 255-267, 1981.

[12] LI, J. Fuzzy evolutionary approaches for bus and rail driver scheduling. Tese (Doutorado em Ciência da Computação) - University of Leeds, England, 2002.

[13] MAURI, G. R. Novas heurísticas para o problema de escalonamento de tripulações. Dissertação (Mestrado em Computação Aplicada) - Instituto Nacional de Pesquisas Espaciais, São José dos Campos, 2004.

[14] KWAN, A. S. K., KWAN, R. S. K.; WREN, A. Driver scheduling using genetic algorithms with embedded combinatorial traits. In: INTERNATIONAL CONFERENCE ON COMPUTER-AIDED SCHEDULING OF PUBLIC TRANSPORT. Proceeding.... Boston: MIT, ano1997. p. 81-102

[15] CASTRO, J. R. P. Geração de escalas de trabalho em transporte urbano de passageiros: uma formulação heurística para a formulação do problema de cobertura de conjuntos. Dissertação (Mestrado em Engenharia de Produção) - Universidade Federal de Santa Catarina, Florianópolis, 2002. 
[16] FORSYTH, P.;WREN, A. An ant system for bus driver scheduling. In: International Workshop on Computer-Aided Scheduling of Public. Proceeding... Boston: MIT, 1997 p. 405-421.

[17] LOURENÇO, H. R., PAIXÃO, J.; PORTUGAL, R. Multiobjective metaheuristics for the bus-driver scheduling problem. Transportation Science, Atlanta, GA, v. 35, n. 3, p. 331-343, 2001.

[18] SHEN, Y.; e KWAN, R. S. K. Tabu search for driver scheduling. In: Yindong Shen, Raymond S. K. Kwan, Computer-Aided Scheduling of Public Transport, Berlin, Springer-Verlag, 2001, p. 121-135.

[19] FORES, S. Column generation approaches to bus driver scheduling. Tese (Doutorado em Ciência da Computação) - University of Leeds, Leeds, 1996.

[20] GIBBONS, A. Algorithmic graph theory. New York: Cambridge University, 1985.

[21] KIRKPATRICK, S.; GELATT JR., C. D.; VECCHI, M. P. Optimization by Simulated Annealing. Science, Washington, v. 220, n. 4598, p. 671-683, 1983.

[22] CERNY, V. Thermodynamical approach to the Traveling Salesman Problem. Journal of Optimization Theory and Applications, New York, US, 45, p. 41-51, 1985.

[23] JACOBS, L. W.; BRUSCO, M. J. A Local-Search Heuristic for Large Set-Covering Problems. Naval Search Logistics, Malden, v. 42, n. 7, p. 1129-1140, 1995.

[24] CASTRO, E. C.; CONSTANTINO, A. A. Escalonamento de veículos e condutores em empresas de transporte urbano rodoviário. Trabalho conclusão de curso (Graduação em Informática). Universidade Estadual de Maringá, Maringá, 2003.

[25] COnStantino, A. A.; REIS, P. A. MENDONÇA, C. F. X.; FIGUEIREDO, M. F. Aplicação de algoritmos genéticos ao problema de cobertura de conjunto. -In: SIMPÓSIO BRASILEIRO DE PESQUISA OPERACIONAL, 35. Anais... Natal: Sobrapo, 2003. 